\title{
Improving Energy Efficiency of Smart Household Appliances and Devices
}

\author{
Viktor I. Suzdorf, Aleksandr S. Meshkov \\ Komsomolsk-on-Amur State Technical University, Khabarovsk, Russian Federation \\ Email: susdorf@mail.ru
}

Received July 2013

\begin{abstract}
With the ever-increasing volume of applications of various kinds of electric drives in all spheres of human activity, the issues in improving the efficiency of the electromechanical converters of electric energy, one of the most important components of the electric drive (ED), are becoming increasingly important. Such issues include reducing their weight and size, improving the functional characteristics of these devices to increase their operational life and reducing the cost of manufacture. Taking full advantage of these opportunities relates to the AC and DC single-phase commutator motor (SCM), which is widely used in regulated and high-speed motor drives in home appliances and electric hand tools. The SCM is used in machinery where the load torque has a hyperbolic dependence on the rotational speed and the need to work with a large motor overload due to the "soft" mechanical characteristics of such motors.
\end{abstract}

\section{Keywords}

Smart Household Appliances; Efficiency; Motor Control

\section{Introduction}

\section{The Efficiency of the Single-Phase Commutator Motor (SCM) Control}

Such motors have qualities that cause their widespread use [1]: The overload capacity is greater than that in other motors used in consumer electrics; the performance of the CMS is greater than that of a DC motor with separate excitation; there is no need for a power supply for the field winding; the SCM is smaller for a given performance than DC motors of independent and parallel excitation; reliability is increased due to the large cross-section of the field winding and the small live inter-track; reversing the motor is simple to implement with a split field winding; the loss is reduced by the same amplitude of the ripple voltage as compared to a DC motor with separate excitation, with a pulsating current; they are smaller and lighter than semiconductor electric drive (ED) systems.

Expansion of the volume of applications of this class of electric motor is also largely due to the fact that the collector-brush assemblies are high-frequency converters due to their reliability, weight, size, and cost indicators, and have prospects for further development.

Typically, the control systems of the ED with SCM are adjustable, due to the process requirements imposed 
on the ED. As for electric tools, and other household appliances, the workflow is at a certain optimum speed for each material. This is due to the need for optimization in terms of energy and enhanced durability.

To control the speed of the drive, a chain is introduced into the semiconductor converter, producing an average value for the control voltage. For the motor power from a standard household circuit, the inverter may be arranged such that the DC rectifier follows the AC circuit. Obviously, the scheme's implementation should differ in greater simplicity and energy efficiency.

The problem of minimizing the power loss can be formulated as follows: To find the dependence of $i(t)$ and $v(t)$, ascertain the maximum integral $a=\int_{0}^{T} v(t) d \tau$, for a given value of the integral equation $Q=\int_{0}^{T}\left(i(t)^{2}+k_{n} \cdot i(t)_{e x}^{2}\right) d \tau$ and communications $\int_{0}^{T}\left(i(t)^{2}+k_{n} \cdot \Phi^{2}\right) d \tau \leq Q_{0}$. where $i(t)$ and $v(t)$ are a function of current and speed in relative units of time, respectively; $a$ is the amount of displacement in relative units and $T$ is the time constant of the motor; with $t$ - time in arbitrary units; $Q$-amount of heat liberated in the motor in relative units; $Q_{0}$-maximum allowable amount of heat that can be released into the armature winding during the time $T$, yet without leading to overheating isolation; and a constant that takes into account the heating winding.

The solution of the Lagrange variational calculus is sought from the Euler equations for the intermediate functions:

$$
F=i^{2}-2 \cdot \lambda_{0} \cdot v+2 \cdot \lambda(v+\mu-i \cdot \Phi)
$$

in general is a function:

$$
\frac{i}{\Phi+i \cdot \frac{d \Phi}{d i}}=\frac{\lambda_{0}}{k}-C \cdot e^{k \cdot \tau}
$$

where $\lambda_{0}$ is the constant and $\lambda$ a function of $\tau$.

In the commutator motors, sequentially exciting the reaction cannot be ignored, since the magnetic flux depends on the armature current. The formula for the optimal control output for an arbitrary relationship between the current and the magnetic flux is $F=F(i)$. A good approximation of such a relationship between the magnetic flux and the armature current is given by:

$$
i=a \cdot \Phi+b \cdot \Phi^{q}
$$

where $a+b=1$ and the value $q$ for different engines is in the range $3-7$.

Then the optimal control law is:

$$
\frac{\left(a \cdot \Phi+b \cdot \Phi^{q}\right) \cdot\left(a+b \cdot q \cdot \Phi^{q-1}\right)}{a \cdot \Phi+\frac{b(q+1)}{2} \cdot \Phi^{q}}=C-\lambda_{0} \cdot \tau
$$

For optimal control of sequentially exciting the commutator motors, the problem of the minimum loss for a given performance and time has to be considered.

Equations of state for an unsaturated collector series-wound motor in relative terms is a system where:

$$
\frac{d i}{d \tau}=\frac{1}{\lambda} \cdot[u-i \cdot(v+\delta)]
$$

With reversible points:

$$
\begin{gathered}
\frac{d v}{d \tau}=i^{2} \text { signi }-\mu_{T} \\
\frac{d Q}{d \tau}=i^{2}
\end{gathered}
$$

The coordinates of the current state of the anchor chain $i$, are speed $v$, angle $\alpha$ and the heat loss $Q$, and of the control parameter is the voltage $u$, through which the desired control law. 
The optimal control problem is considered in a controlled ED, that is, when developing the increment rate in the formulation of the minimum loss: a function $i(t)$ is found, which provides a minimum loss of $Q$. Then, if the set of possible optimal laws of $i(t)$ is the simplest $i(t)=i_{0}(t)=$ const, then the solution is:

$$
i_{0}=\sqrt{\frac{\Delta v}{T}+\mu_{T}}
$$

where $\Delta v=\int_{0}^{T} v^{\prime} d \tau$ is the increase in velocity.

When using Pontryagin's maximum principle, in general, the solution of the problem of stabilization can be obtained as follows:

$$
i^{*}(\tau)=\left\{\begin{array}{lll}
+i_{*_{M}} & \text { if } & 0 \leq \tau \leq \tau_{1} \\
\sqrt{\mu_{T}} & \text { if } & \tau_{1} \leq \tau \leq \tau_{2} \\
-i_{*_{M}} & \text { if } & \tau_{2} \leq \tau \leq T
\end{array}\right.
$$

The efficiency of energy processes is usually defined by the ratio of net power or energy expended to. Without causing difficulties in the simplest cases, this rate is inconvenient in some situations, such as when there is a channel in the power transducer elements and a wide range of changes in the shaft servomotor. Imperfection of the criterion of efficiency of energy conversion results in a significant reduction in the effectiveness of technical measures aimed at saving electricity, making it difficult to obtain objective assessments of the quality of different technical solutions.

As a criterion for evaluation of energy efficiency, largely free of the disadvantages presented, we can offer dynamic efficiency, which is defined by:

$$
\eta_{\text {дин }}=\frac{W}{W+\Delta W}=\frac{\int|P(t) d t|}{\int|P(t) d t|+\int|\Delta P(t) d t|}
$$

where $W$ is the energy consumed by the motor during the period of work, $W \cdot \mathrm{h} ; \Delta W$ is the loss of energy during work, $W \cdot \mathrm{h}, P(t)$ is the motor power for the period of work, $\mathrm{W}, \Delta P(t)$ is the loss of power for the period of operation of the engine, $\mathrm{W}$;

$$
\frac{d \alpha}{d \tau}=v
$$

Using this criterion enables the assessment of the energy efficiency of the system, not only in static mode, but also in a dynamic that does not allow the use of efficiency in the traditional form. The advantages of this include the following criteria: choice of the time interval for the estimate (if provided, the integral equation is defined) and the presence of a non-negative power value, thus maintaining the criterion point for any energy flow directions.

Consumption values and power output are determined according to:

$$
\begin{gathered}
P_{\text {el }}(t)=U_{\text {grid }}(t) \cdot I_{\text {motor }}(t) ; \\
P_{\text {mech }}(t)=M_{\text {motor }}(t) \cdot \omega_{\text {motor }}(t) ;
\end{gathered}
$$

where $U_{\text {grid }}(t)$ is rms voltage motor, $\mathrm{V} ; I_{\text {motor }}(t)$ is the rms current of the motor armature, A; $M_{\text {motor }}(t)$ is the mean value of the electromagnetic torque of the motor, $\mathrm{N} \mathrm{m} ; \omega_{\text {motor }}(t)$ is the rms value of the circular engine speed, $1 / \mathrm{s}$;

The energy consumed by the system is determined by the time integration of power consumption. As a result of the integration of the energy, a balance equation is obtained [2]:

$$
\int_{t 1}^{t 2} U(t) \cdot i(t) d t=\int_{t 1}^{t 2} M_{L} \omega d t+J \cdot \frac{\omega_{2}^{2}-\omega_{1}^{2}}{2}+R \cdot \int_{t 1}^{t 2} i^{2}(t) d t+L \cdot \frac{I_{2}^{2}-I_{1}^{2}}{2}
$$

or

$$
A_{E L}=A_{M E C H}+E_{K I N}+A_{H}+E_{M}
$$


where, $t_{1}, t_{2}$ is the start and end of the integration step; $\omega_{1}, I_{1}$ is the speed and motor current at the beginning of the integration area; $\omega_{2}, I_{2}$ is the speed and motor current at the end of the integration range; and $A_{E L}, A_{M E C H}$, $E_{K I N}, A_{H}$ and $E_{M}$ are electrical, mechanical, kinetic, thermal and magnetic energy, respectively.

For the series-wound motor with a linear or close to it, depending on the current, the flow of the excitation energy efficiency of the engine is determined by the expression [3]:

$$
\eta_{W}=\frac{\sqrt[3]{3 \frac{t}{T}+1}-1}{\sqrt[3]{3 \frac{t}{T}+1}+1}
$$

where $T$ is a time constant of the SCM.

Dependence of the dynamic efficiency of the supply voltage and the time is extreme. Therefore, the surface being located in the space of "dynamic efficiency-voltage-electromagnetic torque", will have a certain maximum. Thus, driving the motor to the point of extreme, when the shaft torque is through the formation of the desired signal control PWM converter, provides high energy-saving effects.

Among the requirements for the engine speed before the other dominated by those who brought the process. Processes require that the appliance chooses stable speeds for performance and the quality of processing.

Thus, the obvious is the need to maintain the frequency of rotation at a constant level regardless of the function of the load torque.

To determine the management criterion, consider the structure "controlled converter—-the series-wound motor" in relative terms:

$$
\begin{gathered}
i=\frac{I}{I_{N}}=\sqrt{\mu} ; \mu=\frac{M}{M_{N}}=i^{2} ; v=\frac{\omega}{\omega_{N}} ; u=\frac{U}{U_{N}} ; \\
\mu_{C}=\frac{M_{L}}{M_{N}} ; \tau=\frac{t}{T_{M}} ; T_{M}=\frac{J \cdot \omega_{N}}{M_{N}} ; \rho=\frac{R_{a r m}}{R_{N}}
\end{gathered}
$$

Then the basic equation of motion in the EP relative units is as follows:

$$
\mu=\frac{d v}{d \tau}+\mu_{L}
$$

In optimal control theory, there are two types of drives: acceleration and position. The ED-electric instrument is in the first group and the optimal control of the ED can be summarized as follows: to change the speed of the motor of the value of $v_{1}$ to $v_{2}$ so that, for any time of the transition, the energy consumed by the inverter is minimal it is necessary to ensure maximum dynamic efficiency:

$$
\eta(\tau)=\frac{\int\left(\mu_{c} \cdot v\right) \cdot d \tau}{\int\left(\mu_{c} \cdot v+\Delta P\right) \cdot d \tau}
$$

where $\Delta P$ is this loss in ED: electrical and mechanica: $\Delta P_{E L} \Delta P_{-m e x}$.

The exploration and development of motor control systems must take into account the limitations imposed by the basic coordinates in the process:

- Current limiting $|i| \leq i_{\max }$;

- Heating restriction; $\int_{0}^{T} i^{2} d t \leq Q_{0}$

- Speed limit; $v=v_{\text {onm }}$

- Limited voltage value; $U \leq U_{\max }$

- Limited acceleration $a=a_{\max }$

- Limit on the jerk $j=j_{\max }$;

It is obvious that maximum functionality will be provided under the following condition:

$$
\int\left(\mu_{L} \cdot v+\frac{u^{2}}{\rho}+\Delta P_{M E C H}\right) d \tau \rightarrow \min
$$


Expressing the equation of motion $d \tau$ and replacing the limits of integration, we get:

$$
\int_{0}^{\tau_{t p}}\left(\mu_{L} \cdot v+\frac{u^{2}}{\rho}+\Delta P_{M E C H}\right) d \tau=\int_{v 1}^{v 2} v \cdot\left(\mu_{L}+\frac{u^{2}}{\rho}+\Delta P_{M E C H}\right) \cdot \frac{d v}{\mu-\mu_{L}}
$$

In those cases where $\mu_{L}$ is velocity, the function does not depend explicitly on the time and route; at least a definite integral is achieved with the proviso that at each speed range $v_{1}-v_{2}$ of the motor a minimum integrand is provided:

$$
\frac{d}{d \mu}\left[\frac{\left(v \cdot \mu_{L}+\frac{u^{2}}{\rho}+\Delta P_{M E C H}\right)}{\mu-\mu_{L}}\right]=0
$$

Then, at a constant speed and ignoring mechanical losses, the solution is:

$$
u= \pm \sqrt{v \cdot \rho \cdot \mu+\rho \cdot \Delta P_{M E C H}}= \pm \sqrt{a \cdot \mu}
$$

It should be noted that this control law can also be derived without the use of optimal control methods, and the definition of efficiency. As already mentioned, the efficiency - the ratio of useful power supplied — and the motor current is proportional to the square root of the electrodynamic motor torque. Then, the relative efficiency of the units is determined by the expression:

$$
\eta=\frac{\mu \cdot v}{u \cdot i}=\frac{\mu \cdot v}{u \cdot \sqrt{\mu}}=\sqrt{\mu} \cdot \frac{v}{u}
$$

Expressing voltage, we get the expression:

$$
u=\sqrt{\mu} \cdot \frac{v_{\text {const }}}{\eta_{\max }}
$$

Assuming that the speed is constant, that is, it is stabilizing, and that efficiency is maintained at a constant level, such as its maximum level, then the voltage takes a function of one variable-the electromagnetic torque.

$$
u(\mu)=\sqrt{\mu} \cdot \frac{v_{\text {const }}}{\eta_{\max }}
$$

The last expression confirms the previously derived management characteristic in order to maintain maximum efficiency in the management of single-phase commutator series-wound motors.

\section{References}

[1] Emadi, A. (2004) Energy-Efficient Electric Motors, Third Edition, Revised and Expanded. Illinois Institute of Technology, Chicago.

[2] Krause, P.C., Wasynczuk, O. and Sudhoff, S.D. (2002) Analysis of Electric Machinery and Drive Systems. WileyIEEE Press. http://dx.doi.org/10.1109/9780470544167

[3] Meshkov, A. and Suzdorf, D. (2010) Improving the Energy Efficiency Factor of Low Power Electric Drives. http://www.rusnauka.com 